\title{
ELLIPTIC EQUATIONS IN DIVERGENCE FORM, GEOMETRIC CRITICAL POINTS OF SOLUTIONS, AND STEKLOFF EIGENFUNCTIONS *
}

\author{
G. ALESSANDRINI ${ }^{\dagger}$ AND R. MAGNANINI ${ }^{\ddagger}$
}

\begin{abstract}
The Stekloff eigenvalue problem (1.1) has $a^{\prime}$ countable number of eigenvalues $\left\{p_{n}\right\}_{n=1,2, \ldots}$, each of finite multiplicity. In this paper the authors give an upper estimate, in terms of the integer $n$, of the multiplicity of $p_{n}$, and the number of critical points and of nodal domains of the eigenfunctions corresponding to $p_{n}$.

In view of a possible application to inverse conductivity problems, the result for the general case of elliptic equations with discontinuous coefficients in divergence form is proven by replacing the classical concept of critical point with the more suitable notion of geometric critical point.
\end{abstract}

Key words. eigenvalue problems, geometric properties of elliptic equations, critical points, inverse conductivity problems

AMS subject classifications. 35J25, 35P99, 35C62, 30C15

1. Introduction. In this paper we are concerned with weak solutions of the elliptic equation in divergence form:

$$
\operatorname{div}(A \nabla u)=0 \quad \text { in } \Omega
$$

and especially with Stekloff eigenfunctions, that is, those nontrivial solutions that, for some constant $p$, the Stekloff eigenvalue, satisfy in the weak sense the boundary condition

$$
A \nabla u \cdot \nu=p u \quad \text { on } \Omega
$$

Here $\Omega$ is a simply connected bounded domain in the plane, with Lipschitz boundary $\partial \Omega, \nu$ is the exterior unit normal to $\partial \Omega$, and $A=\left(a_{i j}\right)$ is a $2 \times 2$ symmetric matrix of $L^{\infty}(\Omega)$ coefficients satisfying, for some constant $\lambda, 0<\lambda \leq 1$, the uniform ellipticity condition

$$
\lambda|\xi|^{2} \leq \sum_{i, j=1}^{2} a_{i j}(z) \xi_{i} \xi_{j} \leq \lambda^{-1}|\xi|^{2}, \quad \text { for every } z \in \Omega, \xi \in \mathbb{R}^{2}
$$

Here and in what follows, we use the complex coordinate $z=x+i y$ in the plane.

The study of this eigenvalue problem was started by Stekloff [St] in 1902. In $§ 3$, we recall the definitions of Stekloff eigenvalues and eigenfunctions; a review of their known properties can be found in Bandle [B]. Research on this subject has been mainly devoted to estimates on eigenvalues (see, for instance, [H-P-S] and also [B] for further references). Let us mention in passing that, in connection with applied problems in fluid mechanics, mixed type problems also have been considered (see [F-K]). Typically, 1993.

* Received by the editors February 2, 1993; accepted for publication (in revised form) June 18,

$\dagger$ Dipartimento di Scienze Matematiche, Università di Trieste, Italy.

$\ddagger$ Dipartimento di Matematica, Università di Firenze, Italy. 
in such problems, we assume that (1.1b) holds only on one portion of $\partial \Omega$, whereas on the rest of $\partial \Omega, A \nabla u \cdot \nu=0$ is assumed.

Our main results, which are summarized in Theorem 3.2, consist of estimates on the multiplicities of Stekloff eigenvalues and the numbers of nodal domains and of critical points of Stekloff eigenfunctions.

One up-to-date motivation of our study of the Stekloff eigenvalue problem comes from the so-called inverse conductivity problem: suppose that the coefficient matrix $A$ (the conductivity) is unknown; we wish to determine it, or, more generally, to recover partial information about it from the knowledge of the so-called Dirichlet to Neumann map $\Lambda_{A}$. Here, $\Lambda_{A}: H^{1 / 2}(\partial \Omega) \rightarrow H^{-1 / 2}(\partial \Omega)$ is defined as the operator that maps the Dirichlet data $u_{\mid \partial \Omega}$ for (1.1a) into the corresponding Neumann data $A \nabla u \cdot \nu_{\mid \partial \Omega}$ (see, for instance, [Sy-U], [Sy]). Then it is evident that the Stekloff eigenvalues and the boundary traces of the Stekloff eigenfunctions are exactly the eigenvalues and eigenfunctions of $\Lambda_{A}$. Such traces and eigenvalues can be approximately measured by experiments and could be effectively used as the data of the inversion procedure. For a discussion of a related spectral approach to the inverse conductivity problem, see Gisser, Isaacson, and Newell [G-I-N]. A deeper understanding of the geometrical features of Stekloff eigenfunctions would then be helpful in assessing uniqueness and continuous dependence questions for the inverse conductivity problem and perhaps also in the design of reconstruction algorithms.

In addition to the possible applications to inverse problems, the authors have been inspired by the work of Payne and Philippin [P-P] on questions of symmetry related to the Stekloff eigenvalue problem (see also [A-M2]). In this respect, we mention that some of our results, when restricted to Laplace's equation and to the second Stekloff eigenvalue, have already been presented in [P-P].

The flavor of our results is similar to those of Cheng [C] for the eigenfunctions of the Laplacian on surfaces; however, the methods in the proofs and the specific results are different because of the intrinsic differences between the two eigenvalue problems.

To mention the most apparent peculiarity of Stekloff eigenfunctions, observe that, by the maximum principle, every solution of (1.1a), and thus every Stekloff eigenfunction, can have neither interior maxima nor minima, and each of its level sets must reach the boundary. Due to this observation, the geometric-topologic properties of level lines and level sets of Stekloff eigenfunctions are quite different from those of the vibrating membrane problems. In fact, in some respects, the study of such properties is perhaps easier for equations like (1.1a), for which we have theorems that permit us to estimate the number of interior critical points in terms of the boundary data $[\mathrm{A}]$, [A-M1]. Theorems like this will be our basic tool, along with simple variations of the fundamental Courant's nodal domain theorem.

Still, in view of the application to the inverse conductivity problem, we have chosen to treat the Stekloff eigenvalue problem when no smoothness assumption is imposed on the coefficients in (1.1a). In fact, there are practical cases when the conductivity coefficients are discontinuous and we are especially interested in determining the discontinuities (see, for instance, $[\mathrm{B}-\mathrm{F}-\mathrm{I}],[\mathrm{P}],[\mathrm{Su}-\mathrm{U}]$ ). This generality causes additional technical difficulties: solutions of (1.1a) need not to be differentiable in the classical sense and thus the notion of critical point must be adapted to this nonsmooth setting. For this reason, we introduce the concept of geometric critical point (see Definition 2.3). Roughly speaking, a point will be called a geometric critical point for a solution $u$ of (1.1a) if it is a critical point with respect to an appropriate (possibly nonsmooth) change of variables that makes $u$ become smooth. We also give the definition of geometric index, which generalizes the notion of multiplicity of a critical 
point (see Definition 2.4).

These definitions are based on the crucial remark that the unique continuation property and a representation theorem also hold for solutions of (1.1a). We have not been able to find in the literature theorems of such a kind for equations like (1.1a), whereas they are well known for equations with smoother coefficients (see [Sch]) and for equations not in divergence form (see [B-N]). Although the method of proof of such results may sound familiar to the experts in quasi-conformal mappings and complex analytic methods, we include our own proofs of the unique continuation property and of representation formulas for solutions of (1.1a) (Theorem 2.1 and Corollary 2.2). We trust that these results might be of some independent interest and that they can find useful applications, especially in the field of inverse problems.

We conclude this introduction by pointing out the following consequence of such results. In $[\mathrm{A}],[\mathrm{A}-\mathrm{M} 1]$, when the coefficients in (1.1a) are smooth, an estimate on the maximum number of interior critical points of a solution $u \in C^{1}(\bar{\Omega}) \cap C^{2}(\Omega)$ of (1.1a) was given in terms of the number of times some boundary data of $u$ changes its sign on $\partial \Omega$ (see [A, Thm. 1.1], [A-M1, Thms. 2.1, 2.2], for details). Theorems 2.7 and 2.8 in this paper generalize the above results to equation (1.1a) with nonsmooth coefficients.

2. Geometric critical points. Throughout this section, $B_{R}(0)$ will denote the disk with radius $R$ centered at $z=0$.

THEOREM 2.1 (representation formula). Let $u \in W^{1,2}(\Omega)$ be a nonconstant solution of (1.1a).

There exists a quasi-conformal mapping $\chi: \Omega \rightarrow B_{1}(0)$ and a real-valued harmonic function $h$ on $B_{1}(0)$ such that

$$
u=h \circ \chi \quad \text { in } \Omega .
$$

The dilatation coefficient $\chi_{\bar{z}} / \chi_{z}$ of $\chi$ is bounded by the constant $(1-\lambda) /(1+\lambda)$.

Proof. By (1.1a), the 1-form $\omega=-\left(a_{12} u_{x}+a_{22} u_{y}\right) d x+\left(a_{11} u_{x}+a_{12} u_{y}\right) d y$ is closed in $\Omega$. Therefore, we can find $v \in W^{1,2}(\Omega)$ such that $d v=\omega$. The function $v$ will be called the stream function associated with $u$, in analogy with the theory of gas dynamics (see, for instance, [B-S]).

In other words, $u$ and $v$ satisfy the following elliptic system:

$$
\nabla v=\left(\begin{array}{cc}
0 & -1 \\
1 & 0
\end{array}\right) A \nabla u
$$

almost everywhere in $\Omega$. Note that (2.2) is just a special case of the elliptic systems studied by Bers and Nirenberg in [B-N].

By setting $f=u+i v$ and using the standard notation for complex derivatives, (2.2) takes the form

$$
f \bar{z}=\mu f_{z}+\nu \overline{f_{z}}
$$

where

$$
\mu=\frac{a_{22}-a_{11}-2 i a_{12}}{1+a_{11}+a_{22}+a_{11} a_{22}-a_{12}^{2}}, \quad \nu=\frac{1-a_{11} a_{22}+a_{12}^{2}}{1+a_{11}+a_{22}+a_{11} a_{22}-a_{12}^{2}},
$$

and the following estimate can be easily deduced from (1.2)

$$
|\mu|+|\nu| \leq \frac{1-\lambda}{1+\lambda}<1 \quad \text { in } \Omega
$$


Consequently, since $f \in W^{1,2}(\Omega, \mathbb{C})$, then it is a quasi-regular mapping with dilatation bounded by $(1-\lambda) /(1+\lambda)$. Since $f$ is nonconstant, it can be factored as

$$
f=F \circ \chi \quad \text { in } \Omega
$$

where $F$ is a holomorphic function on the disk $B_{1}(0)$ and $\chi: \Omega \rightarrow B_{1}(0)$ is a quasiconformal homeomorphism (see [L-V]). Finally,

$$
\left|\frac{\chi_{\bar{z}}}{\chi_{z}}\right|=\left|\frac{f_{\bar{z}}}{f_{z}}\right| \leq \frac{1-\lambda}{1+\lambda}
$$

Corollary 2.2 (Unique continuation principle). If there exists $z_{0} \in \Omega$ and positive constants $C_{1}, C_{2}, \ldots, C_{N}, \cdots$ such that

$$
\int_{\Omega \cap B_{R}\left(z_{0}\right)}|\nabla u|^{2} d x d y \leq C_{N} R^{N} \quad \forall R>0, \quad \forall N=1,2, \ldots
$$

then $u$ is constant in $\Omega$.

Proof. Let us suppose by contradiction that $u$ is nonconstant. Without loss of generality, we may set $z_{0}=0$ and $u(0)=v(0)=0$.

Note that, by (2.2), the stream function $v$ associated with $u$ satisfies the following equation:

$$
\operatorname{div}\left(\frac{1}{\operatorname{det} A} A \nabla v\right)=0 \quad \text { in } \Omega
$$

in the weak sense. Such an equation satisfies the ellipticity condition (1.2) as well.

The local boundedness estimate [G-T, Thm. 8.17] is applicable to both (1.1a) and (2.5). Thus, by this estimate, the Poincaré inequality, and (2.4), we have

$$
\begin{aligned}
& \max _{B_{R / 2}(0)}\left|u-u_{R}\right|^{2} \leq K C_{N} R^{N}, \\
& \max _{B_{R / 2}(0)}\left|v-v_{R}\right|^{2} \leq K C_{N} R^{N}, \quad \forall N=1,2, \cdots \quad \text { and } \quad \forall R, 0<R<R_{0} .
\end{aligned}
$$

Here $R_{0}=\operatorname{dist}(0, \partial \Omega), K$ is a positive constant depending on $\lambda$ only, and $u_{R}, v_{R}$ denote the mean values on the disk $B_{R}(0)$ of $u, v$, respectively. Since $u(0)=v(0)=0$, we also have $u_{R}^{2}, v_{R}^{2} \leq K C_{N} R^{N}$, and hence

$$
\max _{B_{R / 2}(0)}\left(u^{2}+v^{2}\right) \leq 8 K C_{N} R^{N}, \quad \forall R, 0<R<R_{0}, \quad \forall N=1,2, \ldots
$$

Now, we claim that there exist $\rho, 0<\rho<R_{0}$, a quasi-conformal homeomorphism $\tilde{\chi}$ of $B_{\rho}(0)$ in itself, and a positive integer $M$ such that the quasi-regular mapping $f=u+i v$ can be factored as

$$
f(z)=[\tilde{\chi}(z)]^{M}, \quad|z|<\rho,
$$

where $\tilde{\chi}$ has dilatation bounded by $(1-\lambda) /(1+\lambda)$ and $\tilde{\chi}(0)=0$. This factorization is readily obtained from (2.3), first, by choosing $M$ as the order of the first nontrivial term in the Taylor series for $F-F(\chi(0))$ at $\chi(0)$, and, second, by noticing the local invertibility of the branches of the multivalued function $[F-F(\chi(0))]^{1 / M}$. 
Since $\tilde{\chi}$ is quasi-conformal, we have that $\tilde{\chi}^{-1}$ is Hölder continuous at zero with some exponent $0<\alpha \leq 1$. From (2.6), (2.7), we have that

$$
Q|z|^{2 M / \alpha} \leq|f(z)|^{2} \leq K C_{N}|z|^{N}, \quad \forall z,|z|<\rho, \quad \forall N=1,2, \ldots
$$

for some positive constant $Q$, and this is impossible.

Definition 2.3. We say that $z_{0} \in \Omega$ is a geometric critical point for $u$, if we have $\nabla h\left(\chi\left(z_{0}\right)\right)=0$, where $h$ and $\chi$ are, respectively, the harmonic function and the quasi-conformal mapping appearing in (2.1).

Remark. It is an obvious, but essential, consequence of this definition that geometric critical points of nonconstant solutions of (1.1a) are isolated.

We now recall a classical definition of the index of a smooth function (see [Mi]). For a $C^{1}$ function $h$ with isolated critical points in the disk $B_{1}(0)$ and a subdomain $D \subset \subset B_{1}(0)$ such that $\partial D$ is smooth and contains no critical point of $h$, the index of $h$ in $D$ is

$$
I(D, h)=-\frac{1}{2 \pi} \int_{\partial D} d \arg (\nabla h) .
$$

With such a choice of the sign, if $h$ is harmonic, $I(D, h)$ gives the number of critical points of $h$ in $D$, when counted according to their multiplicities. Moreover, $I(D, h)$ is constant under perturbations of $D$ that contain the same critical points, and its definition can be extended to the case when $\partial D$ is nonsmooth.

We generalize this notion to nonconstant solutions of (1.1a).

DEFINITION 2.4. Let $D \subset \subset \Omega$ be an open set. If $u$ has no geometric critical points on $\partial D$, we define the geometric index of $u$ in $D$ as

$$
I(D, u)=I(\chi(D), h)
$$

where $h$ and $\chi$ are as in Theorem 2.1 .

Moreover, we define the geometric index of $u$ at $z_{0} \in \Omega$ as

$$
I\left(z_{0}, u\right)=\lim _{r \rightarrow 0} I\left(B_{r}\left(z_{0}\right), u\right) .
$$

Such a limit exists, since the geometric critical points of a solution of (1.1a) are isolated.

The next lemma gives a sort of justification for the term "geometric" in the previous definitions and shows that these do not depend on the particular choice of the representation (2.1).

LEMMA 2.5. Let $u$ be a nonconstant solution of (1.1a). If $z_{0} \in \Omega$ is a geometric critical point for $u$ with geometric index $I=I\left(z_{0}, u\right)$, then there exists a neighborhood $U \subset \Omega$ of $z_{0}$ such that the level line $\left\{z \in U: u(z)=u\left(z_{0}\right)\right\}$ is made of $I+1$ simple arcs, whose pairwise intersection consists of $\left\{z_{0}\right\}$ only.

Proof. By the representation (2.1), since $\chi$ is a quasi-conformal homeomorphism, it is enough to look at the level line $\left\{\zeta \in B_{1}(0): h=h\left(\chi\left(z_{0}\right)\right)\right\}$ near $\chi\left(z_{0}\right)$.

Since $I\left(\chi\left(z_{0}\right), h\right)=I\left(z_{0}, u\right)=I$, then $h-h\left(\chi\left(z_{0}\right)\right)$ is asymptotic to a homogeneous harmonic polynomial of degree $I+1$ near $\chi\left(z_{0}\right)$.

Remark. Observe that, if $u$ is $C^{1}$ in a neighborhood of $z_{0}$ (which happens, for instance, when $A$ is Hölder continuous; see [Sch]), then $z_{0}$ is a geometric critical point with geometric index $I$ if and only if $\nabla u\left(z_{0}\right)=0$ with standard index $I$. This is a consequence of Lemma 2.5 above and Lemma 3.1 in [A-M1]. Note that in [A-M1] the opposite sign is chosen in the definition of the index. 
Proposition 2.6 (Continuity of the geometric index). Let $\left\{A_{m}\right\}_{m=1,2, \ldots}$ be a sequence of symmetric matrices with $L^{\infty}(\Omega)$ entries satisfying $(1.2)$. Let $u_{m} \in W^{1,2}(\Omega)$ be weak solutions of

$$
\operatorname{div}\left(A_{m} \nabla u_{m}\right)=0 \quad \text { in } \Omega,
$$

which converge to $u$ in $W_{\mathrm{loc}}^{1,2}(\Omega)$.

If $D \subset \subset \Omega$ is such that $u$ has no geometric critical point on $\partial D$, then we have

$$
\lim _{m \rightarrow \infty} I\left(D, u_{m}\right)=I(D, u) .
$$

Proof. By the proof of Theorem 2.1, for each $u_{m}$ we may construct a stream function $v_{m}$ such that $f_{m}=u_{m}+i v_{m}$ are quasi-regular mappings with dilatation coefficients uniformly bounded by $(1-\lambda) /(1+\lambda)$. By $(2.1)$, we also have $u_{m}=h_{m} \circ \chi_{m}$, and the dilatation coefficients of the quasi-conformal mappings $\chi_{m}$ are also uniformly bounded. Since $u_{m} \rightarrow u$ in $W_{\text {loc }}^{1,2}(\Omega)$, by using the uniform interior bounds for $f_{m}$ and $\chi_{m}$ in $C^{\alpha}$ (see [G-T, Thm. 8.24]), we have that, possibly passing to subsequences, $h_{m}$ and $\chi_{m}$ converge, respectively, in $C_{\mathrm{loc}}^{\infty}\left(B_{1}(0)\right)$ and $C_{\mathrm{loc}}^{0}(\Omega) \cap W_{\mathrm{loc}}^{1,2}(\Omega)$, to the functions $h$ and $\chi$ corresponding to $u$ in the representation (2.1).

By definition, $I(D, u)=I(\chi(D), h)$ and $I\left(D, u_{m}\right)=I\left(\chi_{m}(D), h_{m}\right)$. Furthermore, by our hypothesis, $\nabla h$ does not vanish on $\partial \chi(D)$, so that $\left|\nabla h_{m}\right|$ is uniformly bounded away from zero on $\partial \chi(D)$, for $m$ large enough. Thus,

$$
I(\chi(D), h)=\lim _{m \rightarrow \infty} I\left(\chi(D), h_{m}\right),
$$

and hence, by the $C_{\mathrm{loc}}^{0}(\Omega)$ convergence of $\chi_{m}$, we arrive at (2.8). Observe now that, since the very beginning of our argument, we could have replaced the sequence $\left\{u_{m}\right\}$ with any of its subsequences. Therefore, the limit in (2.8) exists and the stated equality holds.

THEOREM 2.7. Let $g \in H^{1 / 2}(\partial \Omega)$ be of bounded variation on $\partial \Omega$ and such that $\partial \Omega$ can be split into $2 M$ arcs on which alternatively $g$ is a nondecreasing and nonincreasing function of the arclength parameter.

Let $u \in W^{1,2}(\Omega)$ be the unique solution of (1.1a) satisfying the Dirichlet condition $u=g$ on $\partial \Omega$.

Then the geometric critical points of $u$ in $\Omega$, when counted according to their indices, are at most $M-1$.

Proof. In view of Lemma 2.5 above, this is just a rephrasing of Theorem 1.1 in [A1]. We omit the details.

THEOREM 2.8. Let $g \in H^{-1 / 2}(\partial \Omega)$ be such that $\partial \Omega$ can be split into $2 M$ closed arcs $\Gamma_{1}, \ldots, \Gamma_{2 M}$ such that $(-1)^{j} g \geq 0$ on $\Gamma_{j}, j=1, \ldots, 2 M$, in the sense of distributions.

Let $u \in W^{1,2}(\Omega)$ be a solution of (1.1a) satisfying the Neumann condition $A \nabla u$. $\nu=g$ on $\partial \Omega$.

Then, the geometric critical points of $u$ in $\Omega$, when counted according to their indices, are at most $M-1$.

Proof. We may suppose that $\partial \Omega$ is $C^{\infty}$. If it were not so, we could construct a Lipschitz mapping that transforms $\Omega$ into a disk. Such a mapping does not alter the nature of the equation nor the sign conditions on the Neumann data $g$.

Let us choose a sequence $\left\{A_{m}\right\}$ of $C^{\infty}(\bar{\Omega})$ symmetric matrices satisfying (1.2) and such that $A_{m} \rightarrow A$ in $L^{p}(\Omega)$, for some $1 \leq p<\infty$. It is a straightforward exercise now to construct a sequence $\left\{g_{m}\right\} \subset C^{\infty}(\partial \Omega)$ converging to $g$ in $H^{-1 / 2}(\partial \Omega)$ and such 
that $\int_{\partial \Omega} g_{m} d s=0$ and $(-1)^{j} g_{m}>0$ in the interior of each $\Gamma_{j}, j=1, \ldots, 2 M$, for all $m=1,2, \ldots$.

For any integer $m$, let $u_{m} \in C^{\infty}(\bar{\Omega})$ be the unique solution of the following problem:

$$
\operatorname{div}\left(A_{m} \nabla u_{m}\right)=0 \quad \text { in } \Omega, \quad A_{m} \nabla u_{m} \cdot \nu=g_{m} \quad \text { on } \partial \Omega,
$$

such that $\int_{\Omega} u_{m} d x d y=\int_{\Omega} u d x d y$. Since $u_{m}$ is smooth on $\partial \Omega$, we can apply Theorem 2.2 in [A-M1] and obtain $I\left(D, u_{m}\right) \leq M-1$ for every integer $m$.

Moreover, we can easily see that, by possibly passing to subsequences, $u_{m} \rightarrow u$ in $W_{\text {loc }}^{1,2}(\Omega)$, and hence Proposition 2.6 is applicable. Therefore, for any $D \subset \subset \Omega$ such that $\partial D$ does not contain any geometric critical point of $u$, we have $I(D, u)=$ $\lim _{m \rightarrow \infty} I\left(D, u_{m}\right)$, and hence $I(D, u) \leq M-1$. By the arbitrariness of $D$ in $\Omega$, we obtain our thesis.

3. Multiplicity of Stekloff eigenvalues and geometric critical points of Stekloff eigenfunctions. As is well known, by observing that the trace imbedding $W^{1,2}(\Omega) \hookrightarrow L^{2}(\partial \Omega)$ is compact, the Stekloff eigenfunctions and eigenvalues in $W^{1,2}(\Omega)$ are characterized as the critical points and critical values of the Rayleigh quotient

$$
R(u)=\frac{\int_{\Omega} A \nabla u \cdot \nabla u d x d y}{\int_{\partial \Omega} u^{2} d s}
$$

here $d s$ denotes the arclength element on $\partial \Omega$ (see [St]). The $n$th Stekloff eigenvalue $p_{n}$ can be recursively defined as the minimum of the quotient (3.1) over all functions of class $W^{1,2}(\Omega)$ that are orthogonal in $L^{2}(\partial \Omega)$ to the subspaces $V_{k}, k=1, \ldots, n-1$, where

$$
V_{k}=\left\{u \in W^{1,2}(\Omega): u \text { is a weak solution of (1.1) with } p=p_{k}\right\}
$$

In this way, we can form a divergent sequence $0=p_{1}<p_{2}<\cdots<p_{n}<\cdots$ of eigenvalues, each of them of finite multiplicity.

Definition 3.1. Let $p_{n}$ be the nth Stekloff eigenvalue; we denote by $\mu_{n}$ its multiplicity, that is,

$$
\mu_{n}=\operatorname{dim} V_{n}
$$

For $n \geq 2$, we will also set

$$
\kappa_{n}=\max _{u \in V_{n} \backslash\{0\}} \#\left\{\begin{array}{c}
\text { geometric critical points of } u \text { in } \Omega, \\
\text { counted according to their index }
\end{array}\right\} .
$$

A nodal domain $\Omega_{k}$ of $u \in V_{n}$ is a connected component of the set $\{z \in \Omega: u(z) \neq 0\}$, while a connected component of a set $\partial \Omega_{k} \cap \partial \Omega$ will be referred to as a boundary nodal domain of $u$.

We then define

$$
\begin{aligned}
\Delta_{n} & =\max _{u \in V_{n} \backslash\{0\}} \#\{\text { nodal domains of } u\}, \\
\delta_{n} & =\max _{u \in V_{n} \backslash\{0\}} \#\{\text { boundary nodal domains of } u\} .
\end{aligned}
$$


THEOREM 3.2. The following inequalities hold:

$$
\begin{aligned}
\Delta_{n+1} & \leq 1+\sum_{k=1}^{n} \mu_{k}, \quad n=1,2, \ldots \\
\kappa_{n} & \leq \Delta_{n}-2, \quad n=2,3, \ldots, \\
\mu_{n} & \leq 2\left(\kappa_{n}+1\right), \quad n=2,3, \ldots
\end{aligned}
$$

CoROllaRY 3.3. For every integer $n \geq 2$, we have

$$
\begin{aligned}
\mu_{n} & \leq 2 \cdot 3^{n-2} \\
\kappa_{n} & \leq 3^{n-2}-1, \\
\Delta_{n} & \leq 3^{n-2}+1 .
\end{aligned}
$$

Proof. By applying (3.7)-(3.9) we obtain the recurrence relation $\mu_{n+1} \leq 2 \times$ $\sum_{k=1}^{n} \mu_{k}, n=1,2, \ldots$ Since $\mu_{1}=1$, we obtain (3.10); (3.11) and (3.12) then easily follow from (3.8) and (3.7).

Remark. When $n=2,(3.11)$ gives $\kappa_{2}=0$. This provides a different proof of Lemma 3 in [P-P].

The proof of Theorem 3.2 requires the following lemma, which will be proved at the end of this section.

Lemma 3.4. Let $\Delta_{n}$ and $\delta_{n}$ be defined as in Definition 3.1. Then

$$
\delta_{n} \leq 2\left(\Delta_{n}-1\right), \quad n=2,3, \ldots
$$

Proof of Theorem 3.2. Step 1. We prove (3.7) by contradiction. This argument has been used already in $[\mathrm{K}-\mathrm{S}]$ for the case of the Laplace operator. Suppose there exists a nontrivial eigenfunction $u \in V_{n+1}$ with $\Delta$ nodal domains $\Omega_{1}, \Omega_{2}, \ldots, \Omega_{\Delta}$, and $\Delta \geq 2+\sum_{k=1}^{n} \mu_{k}$. Let us denote by $u_{1}^{(k)}, \ldots, u_{\mu_{k}}^{(k)}$ a basis of the vector space $V_{k}, 1 \leq k \leq n$.

Now consider the function $v=\sum_{j=1}^{\Delta-1} \alpha_{j}\left(u \mathbf{1}_{\Omega_{j}}\right)$; here $\mathbf{1}_{\Omega_{j}}$ denotes the characteristic function of the set $\Omega_{j}$. The real numbers $\alpha_{1}, \ldots, \alpha_{\Delta-1}$ can be chosen not all zero and such that

$$
\int_{\partial \Omega} v u_{\ell}^{(k)} d s=0, \quad \text { for all } \ell=1, \ldots, \mu_{k}, \quad k=1,2, \ldots, n
$$

in fact (3.14) provides $\sum_{k=1}^{n} \mu_{k} \leq \Delta-2$ linear homogeneous conditions on $\Delta-1$ parameters.

In view of (3.14), the function $v$ is admissible for the variational characterization (3.1) of $p_{n+1}$. From the definition of $v$, we have

$$
\begin{aligned}
\int_{\Omega_{j}} A \nabla v \cdot \nabla v d x d y & =\int_{\partial \Omega_{j}}(A \nabla v \cdot \nu) v d s \\
& =\alpha_{j}^{2} \int_{\partial \Omega_{j}}(A \nabla u \cdot \nu) u d s, \quad j=1, \ldots, \Delta-1 .
\end{aligned}
$$

Hence, (1.1b) implies

$$
\int_{\Omega_{j}} A \nabla v \cdot \nabla v d x d y=p_{n+1} \alpha_{j}^{2} \int_{\partial \Omega_{j}} u^{2} d s, \quad j=1, \ldots, \Delta-1
$$


and, by adding the above $\Delta-1$ relations, we obtain

$$
\int_{\Omega} A \nabla v \cdot \nabla v d x d y=p_{n+1} \int_{\partial \Omega} v^{2} d s
$$

Therefore, $v$ is a nontrivial Stekloff eigenfunction corresponding to the eigenvalue $p_{n+1}$. Now, since $v \equiv 0$ on $\Omega_{\Delta}$, by the unique continuation property, we have $v \equiv 0$ on $\Omega$, which is a contradiction.

Step 2. Let $u_{n} \in V_{n}$; by (1.1b) and by Lemma 3.4, $A \nabla u_{n} \cdot \nu$ satisfies the hypotheses of Theorem 2.7 with $M \leq \Delta_{n}-1$; thus, (3.8) follows easily.

Step 3. By contradiction, suppose $\mu_{n} \geq 2\left(\kappa_{n}+1\right)+1$.

Let $u^{(j)}, j=1, \ldots, \mu_{n}$, be a basis of $V_{n}$ and fix $\kappa_{n}+1$ distinct points $z_{1}, \ldots, z_{\kappa_{n}+1}$ in $\Omega$. As we did in the proof of Theorem 2.8, we approximate $A$ by a sequence of matrices $\left\{A_{m}\right\}_{m=1,2, \cdots}$ with $C^{\infty}(\Omega)$-coefficients satisfying (1.2). For each $j, 1 \leq j \leq \mu_{n}$, the weak solutions $u_{m}^{(j)}$ of the Dirichlet problem

$$
\operatorname{div}\left(A_{m} \nabla u_{m}^{(j)}\right)=0 \quad \text { in } \Omega, \quad u_{m}^{(j)}-u^{(j)} \in W_{0}^{1,2}(\Omega),
$$

are $C^{\infty}(\Omega)$-functions and form a sequence $u_{m}^{(j)}$ that converges to $u^{(j)}$ in $W_{\text {loc }}^{1,2}(\Omega)$. By our hypothesis on $\mu_{n}$, for each $j, 1 \leq j \leq \mu_{n}$, we can find real numbers $\alpha_{m}^{(j)}$ such that $\sum_{j=1}^{\mu_{n}}\left(\alpha_{m}^{(j)}\right)^{2}=1, m=1,2, \cdots$ and, also

$$
\sum_{j=1}^{\mu_{n}} \alpha_{m}^{(j)} \nabla u_{m}^{(j)}\left(z_{\ell}\right)=0, \quad \text { for all } \ell=1, \ldots, \kappa_{n}+1, \quad m=1,2, \ldots
$$

For each $j=1, \ldots, \mu_{n}$, the sequence $\alpha_{m}^{(j)}$ can be chosen to converge to some number $\alpha^{(j)}$ so that we have

$$
\sum_{j=1}^{\mu_{n}}\left(\alpha^{(j)}\right)^{2}=1
$$

Now, let $D$ be an open set with $\bar{D} \subset \Omega, z_{1}, \ldots, z_{\kappa_{n}+1} \in D$, and such that $\partial D$ does not contain any geometric critical point of the function $v=\sum_{j=1}^{\mu_{n}} \alpha^{(j)} u^{(j)}$. The sequence of functions $v_{m}=\sum_{j=1}^{\mu_{m}} \alpha_{m}^{(j)} u_{m}^{(j)}$ is such that $I\left(v_{m}, D\right) \geq \kappa_{n}+1$, by (3.15). Moreover, by possibly passing to a subsequence, $v_{m} \rightarrow v$ in $W_{\mathrm{loc}}^{1,2}(\Omega)$, as $m \rightarrow \infty$, so that Proposition 2.6 implies that $I(v, D) \geq \kappa_{n}+1$, that is, $v$ is a nontrivial eigenfunction in $V_{n}$ with at least $\kappa_{n}+1$ geometric critical points in $D \subset \Omega$. This is a contradiction.

We conclude by giving a sketch of the proof of Lemma 3.4. To this end, we introduce the following definitions.

Definition 3.5. We say that a simply connected open subset $A$ of $\Omega$ is a cap, if $\partial \Omega \cap \partial A$ is connected and nonempty.

Let $\Omega_{1}, \ldots, \Omega_{K}$ be open subsets of $\Omega$; we say that $\left\{\Omega_{k}\right\}_{k=1, \ldots, K}$ is an admissible covering of $\Omega$, if $\Omega_{1}, \ldots, \Omega_{K}$ are pairwise disjoint, $\Omega \subset \bigcup_{k=1}^{K} \bar{\Omega}_{k}$, and $\partial \Omega_{k} \cap \partial \Omega \neq \emptyset$, for every $k=1, \ldots, K$.

Proof of Lemma 3.4 (Sketch). Let $u \in V_{n}, u$ nontrivial, and let $\Omega_{1}, \ldots, \Omega_{K}$ be the nodal domains of $u$ in $\Omega$; these sets form an admissible covering of $\Omega$.

Now, let $N_{\Omega}$ be the number of boundary nodal domains of $u$ in $\partial \Omega$. Then (3.13) is implied by $N_{\Omega} \leq 2(K-1)$. This inequality is readily proved by induction on the 
number $K$ of nodal domains and by using the following facts, the proofs of which are straightforward:

(i) Every nodal domain $\Omega_{k}$ is simply connected.

(ii) The covering $\left\{\Omega_{k}\right\}_{k=1, \ldots, K}$ contains at least one cap.

(iii) If $\Omega_{K}$ is a cap, then $\tilde{\Omega}=\Omega \backslash \bar{\Omega}_{K}$ is a simply connected open set and $\left\{\Omega_{k}\right\}_{k=1, \ldots, K-1}$ is an admissible covering of $\tilde{\Omega}$. Moreover, $N_{\Omega} \leq N_{\tilde{\Omega}}+2$.

\section{REFERENCES}

[A] G. Alessandrini, Critical points of solutions of elliptic equations in two variables, Ann. Scuola Norm. Sup. Pisa Cl. Sci. (4), 14 (1987), pp. 229-256.

[A-M1] G. AlESSANDRINI AND R. MAGNANINI, The index of isolated critical points and solutions of elliptic equations in the plane, Ann. Scuola Norm. Sup. Pisa Cl. Sci. (4), 19 (1992), pp. $567-589$.

[A-M2] - Symmetry and non-symmetry for the overdetermined Stekloff eigenvalue problem, Z. Angew. Math. Phys., 45 (1994), pp. 44-52.

[B] C. BANDle, Isoperimetric Inequalities and Applications, Pitman, London, 1980.

[B-F-I] H. Bellout, A. Friedman, ANd V. Isakov, Stability for an inverse problem in potential theory, Trans. Amer. Math. Soc., 332 (1992), pp. 271-296.

[B-S] S. Bergman AND M. Schiffer, Kernel Functions and Differential Equations in Mathematical Physics, Academic Press, New York, 1953.

[B-N] L. BERS AND L. NIRENBERG, On a representation theorem for linear elliptic systems with discontinuous coefficients and its applications, in Convegno Internazionale sulle Equazioni Lineari alle Derivate Parziali, Cremonese, Roma 1955.

[C] S-Y. Cheng, Eigenfunctions and nodal sets, Comment. Math. Helv., 51 (1976), pp. 43-55.

[F-K] D. W. Fox And J. R. Kuttler, Sloshing frequencies, Z. Angew. Math. Phys., 34 (1983), pp. 668-696.

[G-I-N] D. G. Gisser, E. IsAaCson, AND J. C. Newell, Electric current computed tomography and eigenvalues, SIAM J. Appl. Math., 50 (1990), pp. 1623-1634.

[G-T] D. Gilbarg and N. S. Trudinger, Elliptic Partial Differential Equations of Second Order, Springer-Verlag, Berlin, 1983.

[H-P-S] J. Hersch, L. E. PAYNe, AND M. M. Schiffer, Some inequalities for Stekloff eigenvalues, Arch. Rational Mech. Anal., 57 (1974-75), pp. 99-114.

[I] V. IsAKov, On uniqueness of recovery of a discontinuous conductivity coefficient, Comm. Pure Appl. Math., 41 (1988), pp. 865-877.

[K-S] J. R. Kuttler AND V. G. Sigillito, An inequality for a Stekloff eigenvalue by the method of defect, Proc. Amer. Math. Soc., 20 (1969), pp. 357-360.

[L-V] O. Lehto AND K. VIRTANen, Quasiconformal Mappings in the Plane, Springer-Verlag, Berlin, 1973.

[Mi] J. Milnor, Differential Topology, Princeton University Press, Princeton, NJ, 1958.

[P-P] L. E. PAYNe AND G. A. PhilipPIn, Some overdetermined boundary value problems for harmonic functions, Z. Angew. Math. Phys., 42 (1991), pp. 864-873.

[P] J. PowelL, On a small perturbation in the two dimensional inverse conductivity problem, J. Math. Anal. Appl., to appear.

[Sch] F. Schulz, Regularity Theory for Quasilinear Elliptic Systems and Monge-Ampère Equations in Two Dimensions, Springer-Verlag, New York, 1990.

[St] M. W. Stekloff, Sur les problèmes fondamentaux en physique mathématique, Ann. Sci. Ecole Norm. Sup., 19 (1902), pp. 455-490.

[Su-U] Z. SUN AND G. UHLMANN, Recovery of singularities for formally determined inverse problems, Comm. Math. Phys., 153 (1993), pp. 431-451.

[Sy] J. SYLVESTER, An anisotropic inverse boundary value problem, Comm. Pure Appl. Math., 43 (1990), pp. $201-233$.

[Sy-U] J. Sylvester AND G. Uhlmann, A uniqueness theorem for an inverse boundary value problem, Ann. of Math., 125 (1987), pp. 153-169. 